\title{
Comparison of Korean vs. American Thyroid Imaging Reporting and Data System in Malignancy Risk Assessment of Indeterminate Thyroid Nodules
}

\author{
Sunyoung Kang ${ }^{1,2,3}$, Seul Ki Kwon ${ }^{1,2,3}$, Hoon Sung Choi ${ }^{4}$, Min Joo Kim ${ }^{1}$, Young Joo Park ${ }^{1,2}$, Do Joon Park ${ }^{1,2}$, \\ Sun Wook Cho ${ }^{1,2}$ \\ ${ }^{1}$ Department of Internal Medicine, Seoul National University College of Medicine; ${ }^{2}$ Department of Internal Medicine, Seoul \\ National University Hospital, Seoul; ${ }^{3}$ Department of Internal Medicine, Uijeongbu Eulji Medical Center, Eulji University \\ School of Medicine, Uijeongbu; ${ }^{4}$ Department of Internal Medicine, Kangwon National University School of Medicine, \\ Chuncheon, Korea
}

Background: The management of cytologically indeterminate thyroid nodules is challenging for clinicians. This study aimed to compare the diagnostic performance of the Korean Thyroid Imaging Reporting and Data Systems (K-TIRADS) with that of the American College of Radiology (ACR)-TIRADS for predicting the malignancy risk of indeterminate thyroid nodules.

Methods: Thyroid nodules diagnosed by fine-needle aspiration (FNA) followed by surgery or core needle biopsy at a single referral hospital were enrolled.

Results: Among 200 thyroid nodules, 78 (39.0\%) nodules were classified as indeterminate by FNA (Bethesda category III, IV, and $\mathrm{V})$, and $114(57.0 \%)$ nodules were finally diagnosed as malignancy by surgery or core needle biopsy. The area under the curve (AUC) was higher for FNA than for either TIRADS system in all nodules, while all three methods showed similar AUCs for indeterminate nodules. However, for Bethesda category III nodules, applying K-TIRADS 5 significantly increased the risk of malignancy compared to a cytological examination alone $(50.0 \%$ vs. $26.5 \%, P=0.028)$, whereas applying ACR-TIRADS did not lead to a change.

Conclusion: K-TIRADS and ACR-TIRADS showed similar diagnostic performance in assessing indeterminate thyroid nodules, and K-TIRADS had beneficial effects for malignancy prediction in Bethesda category III nodules.

Keywords: Thyroid imaging reporting and data systems; Thyroid neoplasms; Thyroid nodule; Thyroid ultrasound; Thyroid guidelines

\section{INTRODUCTION}

Ultrasonography (US) is the first-line imaging modality for the diagnosis of thyroid nodules. Currently, major guidelines rec- ommend fine-needle aspiration (FNA), which is an essential diagnostic tool for assessing the malignancy risk of thyroid nodules, based on US imaging characteristics [1]. After FNA, it is recommended that cytologic results should be reported accord-
Received: 27 July 2021, Revised: 24 August 2021, Accepted: 6 September 2021

Corresponding author: Sun Wook Cho

Department of Internal Medicine, Seoul National University Hospital,

101 Daehak-ro, Jongno-gu, Seoul 03080, Korea

Tel: +82-2-2072-4761, Fax: +82-2-762-2292, E-mail: swchomd@snu.ac.kr
Copyright $\odot 2021$ Korean Endocrine Society

This is an Open Access article distributed under the terms of the Creative Commons Attribution Non-Commercial License (https://creativecommons.org/ licenses/by-nc/4.0/) which permits unrestricted non-commercial use, distribution, and reproduction in any medium, provided the original work is properly cited. 
ing to the Bethesda System for Reporting Thyroid Cytopathology, and further management is chosen based on the results of cytology [2]. However, the reported risks of malignancy in each cytology category vary across studies, especially for indeterminate nodules [2]. Thus, adding US findings has been recommended as an additional diagnostic tool for assessing the malignancy risk of indeterminate thyroid nodules [1-6].

US risk classification systems have been established by several societies: the American Thyroid Association (ATA) [1], the American College of Radiology (ACR) [7], the European Thyroid Association [8], and the Korean Society of Thyroid Radiology [9]. Several recent studies have compared the diagnostic performance of different US risk stratification systems, including a comparison of the diagnostic value of three different Thyroid Imaging Reporting and Data Systems (TIRADS): the Korean, European, and ACR-TIRADS [10]. The Korean-TIRADS (K-TIRADS) showed the best specificity, while the ACR-TIRADS presented the best sensitivity. Meanwhile, another study showed that the ACR-TIRADS outperformed the K-TIRADS in terms of a higher area under the curve (AUC) and lower falsenegative rate [11].

In recent years, several changes have been made in the cytopathologic diagnosis of thyroid nodules. First, the entity of noninvasive follicular thyroid neoplasm with papillary-like nuclear features (NIFTP), which shows an excellent prognosis and an extremely low risk of adverse outcomes, has been introduced [12]. It is mainly placed in Bethesda cytology categories III, IV, and V [13], and a meta-analysis showed that adjusting for NIF$\mathrm{TP}$ as a benign disease significantly reduced the risk of malignancy in categories II to V [14]. Second, a subclassification of atypia (cytologic versus architectural) has been suggested to refine the risk of malignancy in category III nodules by the 2017 Bethesda system [2]. Studies have shown that atypia of undetermined significance or follicular lesion of undetermined significance (AUS/FLUS) nodules with cytologic atypia have a significantly higher risk of malignancy than those with architectural atypia $[15,16]$.

Therefore, it is worthwhile to re-evaluate the diagnostic performance of US system based on the recently changed cytopathologic diagnostic system. This study aimed to compare the diagnostic performance of the K-TIRADS and ACR-TIRADS in terms of predicting the risk of malignancy in each cytology category of the Bethesda system, especially focusing on indeterminate nodules (Bethesda cytology categories III, IV, and V).

\section{METHODS}

\section{Study population}

A total of 1,153 consecutive thyroid nodules from 854 patients (female, 79.9\%; mean age, 53.6 \pm 13.1 years) who underwent FNA at the Department of Endocrinology of Seoul National University Hospital from January to December 2017 were retrospectively screened. Thyroid nodules from patients aged 20 to 85 years that were pathologically confirmed by core needle biopsy (CNB) or surgery were included. Finally, 200 thyroid nodules from 160 patients were enrolled. This study was approved by the Institutional Review Board of Seoul National University Hospital (IRB No. 1911-039-1076). Informed consent was waived because of the retrospective nature of the study and the analysis used anonymous clinical data.

\section{Ultrasonography risk stratification}

A high-resolution US scan using a 5 to $12 \mathrm{MHz}$ linear-array transducer (Philips Affiniti 50 G, Philips Ultrasound Inc., Bothell, WA, USA) was used. After enrollment, US images of all thyroid nodules were retrospectively reviewed and scored using two different systems - the K-TIRADS and ACR-TIRADSby two experienced endocrinologists, who were blinded to patients' clinical information and the pathology results. If there was any discrepancy in scoring, two endocrinologists discussed the issue and adjusted the score.

ACR-TIRADS is composed of five categories as previously described [7], and the TIRADS levels are determined according to the total score from all five categories: highly suspicious (TR5), $\geq 7$ points; moderately suspicious (TR4), 4-6 points; mildly suspicious (TR3), 3 points; not suspicious (TR2), 2 points; benign (TR1), 0 points. The K-TIRADS is presented in the form of a tree diagram and classified nodules into five categories, as previously described [9]: high suspicion, intermediate suspicion, low suspicion, and benign, which are designated as K-TR5, K-TR4, K-TR3, and K-TR2, respectively. To compare the diagnostic performance between K-TIRADS and ACR-TIRADS in each cytology category, malignancy risk was assessed using the combined results of cytology (the six categories of the Bethesda system [2]) and US pattern.

\section{US-guided FNA or CNB procedures and management of cytologically indeterminate nodules}

According to the standard practice protocol of Seoul National University Hospital, FNA was performed according to the indications recommended in the K-TIRADS scoring system [9]. 
Repeated FNA or CNB was considered in nodules with non-diagnostic or indeterminate cytology (Bethesda cytology category III or IV) with highly suspicious US features. Of 1,153 total thyroid nodules, $101(8.8 \%)$ and seven $(0.6 \%)$ nodules were identified as Bethesda category III and IV, respectively. Supplemental Fig. S1 showed the clinical course of these patients. Among the 101 nodules in Bethesda category III, 3 and 55 nodules underwent repeated FNA and CNB, respectively. Among the 55 CNB performed thyroid nodules, 16 nodules underwent thyroid surgery, and their pathologic results were as follows: seven papillary thyroid carcinoma (PTC); one follicular variant of papillary thyroid carcinoma (FVPTC); three NIFTP; and five follicular adenoma. Nine nodules underwent surgery without repeated FNA or CNB for the following reasons: large nodule size $(n=6)$; the simultaneous presence of other nodules confirmed as malignancy $(n=2)$, and the presence of compressive symptoms $(n=1)$. The cytologic examination of repeated FNA in three patients showed Bethesda cytology category I $(n=1)$ or II $(n=2)$. Other 34 patients performed regular US follow-up without repeated FNA or CNB or direct surgery. Additionally, seven $(0.6 \%)$ nodules were identified as Bethesda category IV, and six of them underwent surgery. Finally, among the 108 nodules diagnosed as Bethesda category III or IV by the first FNA, 56 patients were enrolled in this study.

\section{Statistical analysis}

The clinical characteristics of thyroid nodules were compared using the Student's $t$ test. The chi-square test was used to estimate the malignancy risk in each cytology category and subgroups categorized by combined results of cytology and US patterns (K-TIRADS or ACR-TIRADS). The binominal test was used to evaluate whether the malignancy risk in each cytology category was significantly changed by adding the US pattern (K-TIRADS or ACR-TIRADS). To compare the diagnostic performance between K-TIRADS and ACR-TIRADS, a receiver operating characteristic (ROC) curve was used. Statistical analysis was performed using STATA version 13.1 (StataCorp, College Station, TX, USA) and $P<0.05$ was considered to indicate statistical significance for all tests.

\section{RESULTS}

\section{Clinical and cytopathologic characteristics of thyroid nodules}

The baseline clinical characteristics of the thyroid nodules are presented in Table 1. Of 200 finally diagnosed thyroid nodules, 114 nodules $(57.0 \%)$ were confirmed as malignancies. The age at diagnosis of patients with benign thyroid nodules was younger than that of patients with malignant nodules $(48.3 \pm 13.8$ years vs. $54.9 \pm 14.6$ years, $P<0.001)$. The benign thyroid nodules were, on average, larger than the malignant nodules $(2.1 \pm 1.3$

\begin{tabular}{|c|c|c|c|c|}
\hline Characteristic & Total $(n=200)$ & Benign $(n=86)$ & Malignancy $(n=114)$ & $P$ value \\
\hline Age at diagnosis, yr & $51.2 \pm 14.5$ & $54.9 \pm 14.6$ & $48.3 \pm 13.8$ & 0.001 \\
\hline Female sex & $145(72.5)$ & $68(79.1)$ & $77(67.5)$ & 0.071 \\
\hline Size, cm & $1.7 \pm 1.1$ & $2.1 \pm 1.3$ & $1.4 \pm 0.9$ & $<0.001$ \\
\hline Modality of final diagnosis & & & & $<0.001$ \\
\hline Core needle biopsy & $41(20.5)$ & $37(43.0)$ & $4(3.5)$ & \\
\hline Surgery & $159(79.5)$ & $49(57.03)$ & $110(96.5)$ & \\
\hline \multicolumn{5}{|c|}{ Postoperative histologic subtype } \\
\hline PTC & - & - & 107 (93.9) & \\
\hline FVPTC & - & - & $4(3.5)$ & \\
\hline FTC & - & - & $3(2.6)$ & \\
\hline NIFTP & - & $10(11.6)$ & - & \\
\hline Follicular adenoma & - & $9(10.5)$ & - & \\
\hline Other benign lesion & - & $67(77.9)$ & - & \\
\hline
\end{tabular}


Table 2. Malignancy Risk of Thyroid Nodules Assessed by the Bethesda Classification

\begin{tabular}{lcccc}
\hline & $\begin{array}{c}\text { Total } \\
(n=200)\end{array}$ & $\begin{array}{c}\text { Benign } \\
(n=86)\end{array}$ & $\begin{array}{c}\text { Malignancy } \\
(n=114)\end{array}$ & $\begin{array}{c}\text { Recommended malignancy } \\
\text { risk, } \%\end{array}$ \\
\hline Bethesda category & & & $2(25.0)$ & $1-4$ \\
I (non-diagnostic) & $8(4.0)$ & $6(75.0)$ & $1(2.9)$ & $0-3$ \\
II (benign) & $34(17.0)$ & $33(97.1)$ & $13(26.5)$ & $5-15$ \\
III (AUS/FLUS) & $49(24.5)$ & $36(73.5)$ & $12(37.5)$ & - \\
Cytologic atypia & 32 & $20(62.5)$ & $1(10.0)$ & - \\
Architectural atypia & 10 & $9(90.0)$ & 0 & $15-30$ \\
Hurthle cell atypia & 7 & $7(100.0)$ & $2(28.6)$ & $60-75$ \\
IV (FN/SFN) & $7(3.5)$ & $5(71.4)$ & $7(18.2)$ & $78(97.5)$ \\
V (suspicious for malignancy) & $22(11.0)$ & $2(2.5)$ & $97-99$ \\
VI (malignant) & $80(40.0)$ & & \\
\hline
\end{tabular}

Values are expressed as number (\%). $P$ value for benign vs. malignant nodules.

AUS/FLUS, atypia of undetermined significance or follicular lesion of undetermined significance; FN/SFN, follicular neoplasm or suspicious for a follicular neoplasm.

cm vs. $1.4 \pm 0.9 \mathrm{~cm}, P<0.001)$. One hundred and ten $(96.5 \%)$ malignant nodules were diagnosed by surgery, while 37 (43.0\%) benign nodules were diagnosed by CNB $(P<0.001)$. Among the malignant nodules, PTC, FVPTC, and follicular thyroid carcinoma (FTC) were diagnosed in $93.9 \%, 3.5 \%$, and $2.65 \%$ of cases respectively. Of the benign nodules, $11.6 \%$ and $10.5 \%$ were NIFTP and follicular adenomas, respectively.

\section{Malignancy risk of thyroid nodules assessed by the bethesda classification}

First, the malignancy risk of all nodules was assessed by a cytologic examination using the Bethesda categories. Among the 200 nodules, eight (4.0\%), 34 (17.0\%), 49 (24.5\%), seven (3.5\%), 22 (11.0\%), and 80 (40.0\%) were classified as Bethesda cytology category I, II, III, IV, V, and VI, respectively (Table 2). Furthermore, AUS/FLUS nodules were subclassified according to their atypic features. Among 49 nodules, 32 (65.3\%), 10 (20.4\%), and seven (14.3\%) were subclassified as cytologic, architectural, and the Hurthle cell atypia, respectively (Table 2).

Based on the final pathologic diagnosis by surgery or biopsy, the diagnostic performance for predicting malignancy was $26.5 \%, 28.6 \%, 81.8 \%$, and $97.5 \%$ for Bethesda cytology category III, IV, V, and VI, respectively. The risk of malignancy in category III and V nodules was somewhat higher than those previously reported for the 2017 Bethesda system [2]. Within 49 AUS/FLUS nodules, the risk of malignancy was higher in nodules with cytologic atypia than in those with architectural atypia, but the difference was not statistically significant (37.5\% vs. $10.0 \%, P=0.101)$. Seven nodules of Hurthle cell atypia showed no risk of malignancy at all.

\section{Malignancy risk of thyroid nodules assessed by the two US scoring systems}

Next, the malignancy risk was evaluated by two US scoring systems, K-TIRADS and ACR-TIRADS (Table 3, Supplemental Tables S1, S2). Based on the final pathologic diagnosis, the diagnostic performance of K-TIRADS was $8.7 \%, 36.4 \%$, and $86.2 \%$ for K-TR3, K-TR4, and K-TR5, respectively, and that of ACR-TIRADS was $14.3 \%, 33.3 \%$, and $85.3 \%$ for TR3, TR4, and TR5, respectively. Among all 200 nodules, 37 (18.5\%) nodules showed a discordance in the estimated malignancy risk between K-TIRADS and ACR-TIRADS. The ROC analysis showed that AUC of FNA was higher than that of both US scoring systems ( 0.921 vs. 0.855 for K-TIRADS, $P=0.029$; vs. 0.842 for ACR-TIRADS, $P=0.014$ ) (Fig. 1A). The AUC of K-TIRADS and ACR-TIRADS showed no significant difference overall ( 0.855 vs. $0.842, P=0.332)$ or in each cytologic category. In cytologically indeterminate nodules (Bethesda categories III, IV, and V), the diagnostic performance of FNA, K-TIRADS, and ACR-TIRADS showed no significant differences, with AUCs of $0.731,0.754$, and 0.745 , respectively (Fig. 1B). All enrolled nodules were divided into two groups according to the final diagnostic methods, CNB $(n=41)$ or surgery $(n=159)$, and the diagnostic performance of the two US scoring system was compared in each group. The diagnostic performance was similar between the two TIRADS systems in both the CNB and the 
Table 3. Malignancy Risk of Thyroid Nodules Assessed by Two US Scoring Systems: K-TIRADS and ACR-TIRADS

\begin{tabular}{|c|c|c|c|c|c|c|}
\hline US system & $\begin{array}{l}\text { TIRADs } \\
\text { category }\end{array}$ & Total & Benign & Malignant & $P$ value & $\begin{array}{l}\text { Recommended malignancy } \\
\text { risk, \% }\end{array}$ \\
\hline \multirow[t]{5}{*}{ K-TIRADS } & 2 & 1 & $1(100.0)$ & 0 & $<0.001$ & $<3$ \\
\hline & 3 & 46 & $42(91.3)$ & $4(8.7)$ & & $3-15$ \\
\hline & 4 & 44 & $28(63.6)$ & $16(36.4)$ & & $15-50$ \\
\hline & 5 & 109 & $15(13.8)$ & $94(86.2)$ & & $>60$ \\
\hline & Total & 200 & $86(43.0)$ & $114(57.0)$ & & \\
\hline \multirow[t]{5}{*}{ ACR-TIRADS } & 2 & 12 & $12(100.0)$ & 0 & $<0.001$ & $\leq 2$ \\
\hline & 3 & 28 & $24(85.7)$ & $4(14.3)$ & & $\leq 5$ \\
\hline & 4 & 51 & $34(66.7)$ & $17(33.3)$ & & $5-20$ \\
\hline & 5 & 109 & $16(14.7)$ & $93(85.3)$ & & $\geq 20$ \\
\hline & Total & 200 & $86(43.0)$ & $114(57.0)$ & & \\
\hline
\end{tabular}

Values are expressed as number (\%).

US, ultrasonography; K-TIRADS, Korean Thyroid Imaging Reporting and Data System; ACR-TIRADS, American College of Radiology Thyroid Imaging Reporting and Data System.
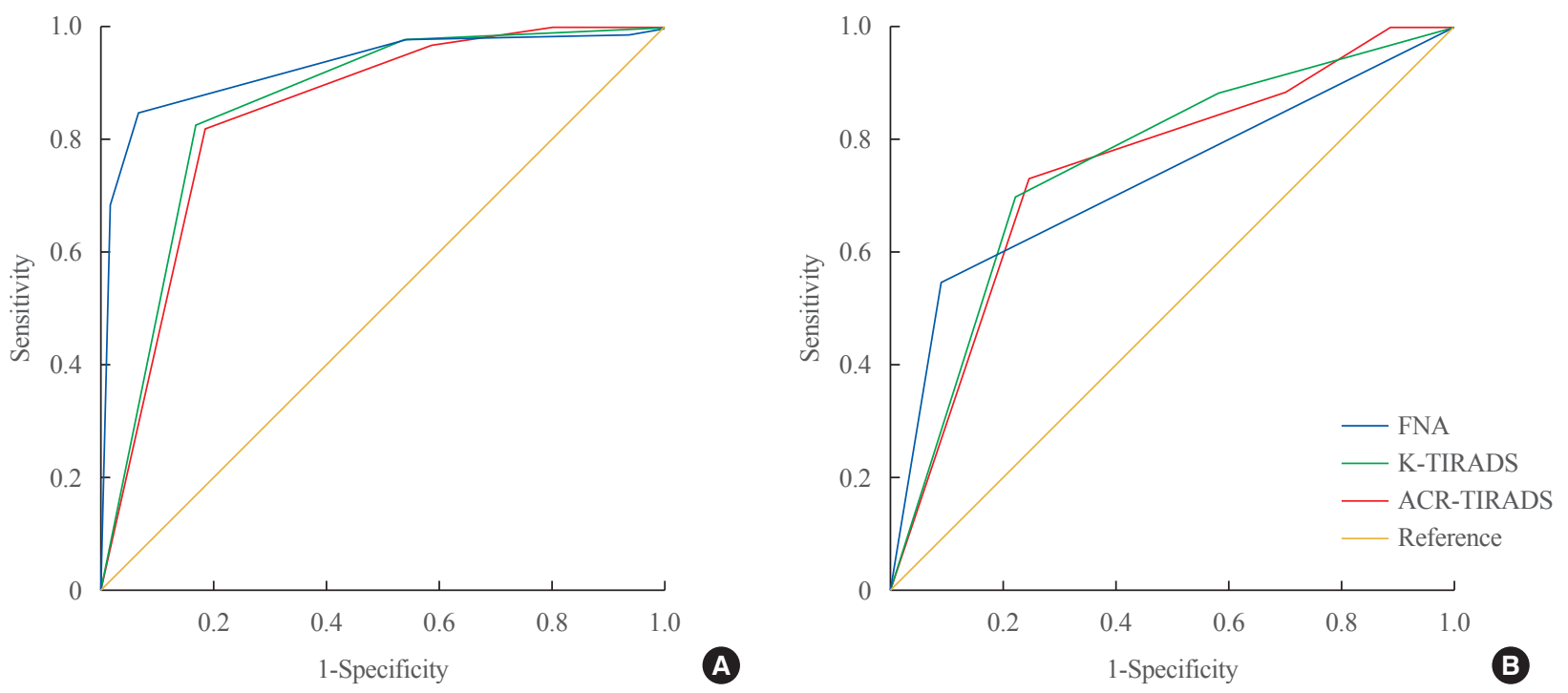

Fig. 1. Receiver operating characteristic curve of Korean Thyroid Imaging Reporting and Data System (K-TIRADS), American College of Radiology Thyroid Imaging Reporting and Data System (ACR-TIRADS), and fine-needle aspiration (FNA) in (A) all thyroid nodules and (B) Bethesda categories III, IV, and V.

surgery groups. The AUC of K-TIRADS versus ACR-TIRADS were 0.865 versus 0.838 in the $\mathrm{CNB}$ group $(P=0.151)$ and 0.900 versus 0.903 in the surgery group $(P=0.819)$.

\section{Effects of US scoring systems on risk assessment of indeterminate thyroid nodules based on FNA}

The diagnostic performance of K-TIRADS and ACR-TIRADS was compared for indeterminate thyroid nodules based on FNA. The sensitivity, specificity, positive predictive value, and nega- tive predictive value were similar between the two systems in each cytology category (Table 4). Next, the predictive values for malignancy in each cytology category were calculated for the combination of FNA results with each US scoring system (Table 5). Interestingly, in nodules of Bethesda category III, adding the US finding of K-TR5 significantly increased the risk of malignancy compared to cytologic findings alone (50.0\% vs. $26.5 \%$, $P=0.028$ ), while adding the US findings of ACR-TIRADS did not (Table 5). Within the nodules of Bethesda category, the pres- 
Table 4. Diagnostic Performance of the Two US Scoring Systems in Each Bethesda Cytology Category

\begin{tabular}{llccccc}
\hline $\begin{array}{l}\text { Bethesda cytology } \\
\text { category }\end{array}$ & \multicolumn{1}{c}{ TIRADS } & Sensitivity, $\%$ & Specificity, $\%$ & PPV, $\%$ & NPV, \% & Accuracy, $\%$ \\
\hline III (AUS/FLUS) & K-TIRADS & $69.2(38.6-90.9)$ & $75.0(57.8-87.8)$ & $50.0(26.0-74.0)$ & $87.1(70.2-96.4)$ & $73.5(58.9-85.1)$ \\
& ACR-TIRADS & $61.5(31.6-86.1)$ & $72.2(54.8-85.8)$ & $44.4(21.5-69.2)$ & $83.9(66.3-94.6)$ & $69.4(54.6-81.8)$ \\
IV (FN/SFN) & K-TIRADS & $0.0(0.0-84.2)$ & $80.0(28.3-99.5)$ & $0.0(0.0-97.5)$ & $66.7(22.3-95.7)$ & $57.1(18.4-90.1)$ \\
& ACR-TIRADS & $0.0(0.0-84.2)$ & $80.0(28.3-99.5)$ & $0.0(0.0-97.5)$ & $66.7(22.3-95.7)$ & $57.1(18.4-90.1)$ \\
V (Suspicious for & K-TIRADS & $77.8(52.4-93.6)$ & $100.0(39.7-100.0)$ & $100.0(76.8-100.0)$ & $50.0(15.7-84.3)$ & $81.8(59.7-94.8)$ \\
malignancy) & ACR-TIRADS & $88.9(65.3-98.6)$ & $100.0(39.7-100.0)$ & $100.0(79.4-100.0)$ & $66.7(22.3-95.7)$ & $90.9(70.8-98.9)$ \\
\hline
\end{tabular}

Values in parentheses are expressed as 95\% confidence interval. For both ACR TIRADS and K-TIRADS, the cut-off value was TIRADS level 5.

US, ultrasonography; PPV, positive predictive value; NPV, negative predictive value; AUS/FLUS, atypia of undetermined significance or follicular lesion of undetermined significance; K-TIRADS, Korean Thyroid Imaging Reporting and Data System; ACR-TIRADS, American College of Radiology Thyroid Imaging Reporting and Data System; FN/SFN, follicular neoplasm or suspicious for a follicular neoplasm.

Table 5. Effects of Each US Scoring System on the Risk Assessment of the Indeterminate Thyroid Nodules Diagnosed by FNA

\begin{tabular}{|c|c|c|c|c|c|c|c|}
\hline \multirow{3}{*}{$\begin{array}{l}\text { Bethesda cytology category } \\
\text { (malignancy risk, \%) }\end{array}$} & \multirow{3}{*}{$\begin{array}{l}\text { TIRADS } \\
\text { category }\end{array}$} & \multicolumn{3}{|c|}{ K-TIRADS } & \multicolumn{3}{|c|}{ ACR-TIRADS } \\
\hline & & \multirow{2}{*}{$\begin{array}{l}\text { Malignancy/ } \\
\text { total }\end{array}$} & \multicolumn{2}{|c|}{ Change in malignancy risk } & \multirow{2}{*}{$\begin{array}{l}\text { Malignancy/ } \\
\text { total }\end{array}$} & \multicolumn{2}{|c|}{ Change in malignancy risk } \\
\hline & & & Interaction $^{\mathrm{a}}$ & $P$ value ${ }^{\mathrm{b}}$ & & Interaction $^{\mathrm{a}}$ & $P$ value $^{\mathrm{b}}$ \\
\hline III (AUS/FLUS) & 2 & - & NA & & $0 / 4(0)$ & No change & 0.292 \\
\hline \multirow[t]{3}{*}{$13 / 49(26.5)$} & 3 & $3 / 18(16.7)$ & No change & 0.257 & $3 / 11(27.3)$ & No change & 0.590 \\
\hline & 4 & $1 / 13(7.7)$ & No change & 0.104 & $2 / 16(12.5)$ & No change & 0.162 \\
\hline & 5 & $9 / 18(50.0)$ & Increase & 0.028 & $8 / 18(44.4)$ & No change & 0.077 \\
\hline Cytologic atypia & 2 & - & NA & & $0 / 3(0)$ & No change & 0.244 \\
\hline \multirow[t]{3}{*}{$(37.5)$} & 3 & $2 / 9(22.2)$ & No change & 0.282 & $2 / 4(50.0)$ & No change & 0.481 \\
\hline & 4 & $1 / 8(12.5)$ & No change & 0.135 & $2 / 10(20.0)$ & No change & 0.211 \\
\hline & 5 & $9 / 15(60.0)$ & No change & 0.065 & $8 / 15(53.3)$ & No change & 0.159 \\
\hline Architectural atypia & 2 & - & NA & & - & NA & \\
\hline \multirow[t]{3}{*}{$(10.0)$} & 3 & $1 / 5(20.0)$ & No change & 0.410 & $1 / 5(20.0)$ & No change & 0.490 \\
\hline & 4 & $0 / 4(0)$ & No change & 0.656 & $0 / 4(0)$ & No change & 0.656 \\
\hline & 5 & $0 / 1(0)$ & NA & & $0 / 1(0)$ & NA & \\
\hline IV (FN/SFN) & 2 & - & NA & & - & NA & \\
\hline \multirow[t]{3}{*}{$2 / 7(28.6)$} & 3 & $1 / 3(33.3)$ & No change & 0.636 & $1 / 2(50.0)$ & No change & 0.490 \\
\hline & 4 & $1 / 3(33.3)$ & No change & 0.636 & $1 / 4(25.0)$ & No change & 0.676 \\
\hline & 5 & $0 / 1(0)$ & NA & & $0 / 1(0)$ & NA & \\
\hline $\mathrm{V}$ (suspicious for malignancy) & 2 & - & NA & & $0 / 1$ & NA & \\
\hline \multirow[t]{3}{*}{$18 / 22(81.8)$} & 3 & $0 / 2(0)$ & Decrease & 0.033 & - & NA & \\
\hline & 4 & $4 / 6(66.7)$ & No change & 0.3 & $2 / 5(40.0)$ & Decrease & 0.045 \\
\hline & 5 & $14 / 14(100)$ & No change & 0.060 & $16 / 16(100)$ & Increase & 0.040 \\
\hline
\end{tabular}

Values are expressed as number (\%).

US, ultrasonography; FNA, fine-needle aspiration; K-TIRADS, Korean Thyroid Imaging Reporting and Data System; ACR-TIRADS, American College of Radiology Thyroid Imaging Reporting and Data System; AUS/FLUS, atypia of undetermined significance or follicular lesion of undetermined significance; NA, not applicable; FN/SFN, follicular neoplasm or suspicious for a follicular neoplasm.

${ }^{a}$ Effect of TIRADS on the malignancy risk of each Bethesda cytology category; ${ }^{b}$ The binominal test was used for the difference of malignancy risk between the combined results of cytology and K-TIRADS and the overall malignancy risk in the same cytology category. 
ence of spiculated margin $(46.2 \%$ vs. $5.6 \%, P=0.001)$, microcalcification $(38.5 \%$ vs. $5.7 \%, P=0.004)$, and non-parallel orientation $(30.8 \%$ vs. $8.3 \%, P=0.048)$ was significantly higher in malignancy than benign, while the presence of hypoechogenity, macro- or rim calcification was not. In nodules of Bethesda category $\mathrm{V}$, adding the US finding of K-TR3 (81.8\% vs. $0 \%$, $P=0.033)$ and ACR-TR4 (81.8\% vs. $40.0 \%, P=0.045)$ significantly decreased the risk of malignancy, whereas adding ACRTR5 significantly increased the risk of malignancy $(81.8 \%$ vs. $100.0 \%, P=0.04)$. There was no additional effect of applying K-TIRADS scores for nodules of Bethesda category IV.

\section{DISCUSSION}

In this study, we demonstrated that two US scoring systemsthe ACR-TIRADS and K-TIRADS - had similar diagnostic performance for indeterminate thyroid nodules diagnosed by FNA. The overall sensitivity and specificity were similar between K-TIRADS and ACR-TIRADS in nodules belonging to Bethesda categories III, IV, and V. However, in nodules belonging to Bethesda category III, adding K-TIRADS 5 significantly increased the risk of malignancy, while adding ACR-TIRADS did not. The presences of spiculated margin, micro-calcification, and non-parallel orientation, were significantly increased in malignant than benign nodules in Bethesda category III. Therefore, the K-TIRADS system may have further beneficial effects in predicting malignancy risk for nodules classified as Bethesda category III.

The management of indeterminate thyroid nodules based on FNA is one of the most challenging topics in the field. Several guidelines recommend repeated FNA and/or lobectomy or molecular diagnostics for indeterminate nodules, but it remains difficult to make an optimal decision because of the wide range of malignancy risk [2]. Thus, several studies have tried to re-assess the malignancy risks of thyroid nodules with indeterminate cytology using various US scoring systems. Recent studies demonstrated that several US scoring systems, including ACR-TIRADS, K-TIRADS, and the ATA guidelines, were useful for refining the malignancy risk of indeterminate thyroid nodules [17-23].

Furthermore, studies have compared the diagnostic performances of different US scoring systems in predicting the malignancy risk of indeterminate thyroid nodules. The present study showed the beneficial effects of applying K-TIRADS on nodules of Bethesda category III in predicting malignancy risk. This result is concordant with that of a recently published study comparing the usefulness of thyroid sonographic risk-stratification systems in the diagnosis of indeterminate or suspicious or unequivocal cytology. In that study, K-TIRADS showed a higher AUC than ACR-TIRADS among AUS/FLUS nodules (0.692 vs. $0.655, P<0.05)$ [24]. The proportion of PTC was high $(79.2 \%)$ in that study, and was even higher in our study $(93.8 \%)$. Since US scoring systems are highly sensitive to PTC, rather than FTC or FVPTC $[25,26]$, K-TIRADS might be more beneficial for diagnosing thyroid cancer in PTC-dominant areas.

Both ACR-TIRADS and K-TIRADS have unique strengths for evaluating the risk of malignancy for thyroid nodules. ACRTIRADS is composed of five categories, including composition, echogenicity, shape, margin, and echogenic foci, and all of those parameters must be evaluated for a final decision [7]. Meanwhile, K-TIRADS is presented in a tree diagram form using two decision steps, wherein malignancy risk is first categorized by echogenicity and solidity, and then by the presence of suspicious US features [9]. Therefore, K-TIRADS is more intuitive and easily applicable in daily clinical settings, while ACR-TIRADS focuses more on accuracy than on ease of application. Since the current evidence, including the present study, shows similar diagnostic performance between these two US scoring systems, an optimal selection needs to be made considering aspects of the real-world environment, including the prevalence of PTC.

The present study was performed in an extremely PTC-dominant area, and $94 \%$ of the histologically confirmed malignant nodules were PTC. Because of its typical cellular morphology [27], we expect that the cytologic diagnosis of PTC is easier than that of FTC or other follicular neoplasms. However, the overall prevalence of AUS/FLUS nodules in this study was $8.8 \%$, similar to that of other countries [28], but not lower than expected. A recent meta-analysis showed an interesting finding comparing Asian and non-Asian populations in AUS/FLUS nodules [28]. In that study, the frequency of AUS/FLUS diagnoses was not significantly different between the Asian and non-Asian cohorts $(8.8 \%$ vs. $9.1 \%, P=0.69)$, while the malignancy risk (43.2\% vs. $26.8 \%$ ), the prevalence of cytologic atypia $(70.3 \%$ vs. $33.5 \%)$, and the proportion of PTC in surgically resected tumors (46.3\% vs. $29.1 \%$ ) of AUS nodules were significantly higher in the Asian studies than the non-Asian studies. The present study showed a higher prevalence of cytologic atypia in AUS/FLUS nodules in association with a high prevalence of PTC. The risk of malignancy was higher in nodules with cytologic atypia than in those with architectural atypia, and adding the US finding of both K-TR5 and ACR-TR5 increased the risk of malignancy compared to cytologic findings alone. 
However, these findings did not obtain statistical significance because of the limited numbers.

The overall malignancy rate of AUS/FLUS nodules in our study was $26.5 \%$, similar to that reported for non-Asian cohorts. This relatively low malignancy risk of AUS/FLUS nodules, compared to the other Asian cohorts, may be explained by the effects of 10 cases of NIFTP, which were included as benign disease, in the present study. Collectively, the present study and a recent meta-analysis [28] showed that the prevalence of AUS/ FLUS nodules were not lower in PTC-dominant Asian cohorts. A further study is needed to explain this intriguing finding.

The present study showed four cases of misdiagnosis. Two of them were false positives and two of them were false negatives. Two nodules initially diagnosed as malignant nodules categorized as Bethesda category VI based on FNA were finally diagnosed as benign hyalinizing trabecular tumor (HTT) after surgery. One comprised hypoechoic and parallel-shaped solid nodules, and was categorized as 4 in both K-TIRADS and ACR-TIRADS. The other one showed the same features, but also had an irregular margin, and was categorized as 5 in K-TIRADS and 4 in ACR-TIRADS. HTT is known to mimic medullary thyroid carcinoma or PTC on cytology, which can lead to the misdiagnosis of these benign tumors as malignancies [29-31]. Jang et al. [32] reported that the most common US features of 12 cases of HTT were hypo- or marked hypo-echogenicity (83.4\%), absence of calcification (91.7\%) and parallel shape (100.0\%), which are consistent with our cases. Further study is needed to identify ways to prevent false-positive diagnoses in cases of HTT.

On the contrary, two cases were categorized as non-diagnostic at initial FNA, but finally diagnosed as malignancies after CNB. Both nodules showed suspicious US features on initial FNA (hypo-echogenicity and a solid component). One case had rim calcification and the other one had both micro- and macro-calcifications. Although the risk of malignancy of initially reported non-diagnostic thyroid nodules is not high (5\% to $10 \%)$ [1], nodules with macro-calcification have a high likelihood of being categorized as non-diagnostic on FNA [33]. In these cases, repeated $\mathrm{CNB}$ can be helpful to make an accurate diagnosis.

There are several limitations in our study. First, we included patients who ultimately underwent CNB or surgery for pathologic confirmation, which means the nodules posed relatively high concerns of malignancy risks. Therefore, our data may overestimate the risk of malignancy. Second, the small sample size may explain the non-significant changes in malignancy risk shown in Table 4. Further research with a larger sample size will be needed to compare different US scoring systems.

In conclusion, both ACR-TIRADS and K-TIRADS had similar diagnostic performance for assessing the malignancy risk of indeterminate thyroid nodules, and K-TIRADS showed beneficial effects on malignancy prediction for nodules belonging to Bethesda cytology category III. Therefore, K-TIRADS may be useful in assessing the malignancy risk of cytologically indeterminate nodules in PTC-prevalent areas.

\section{CONFLICTS OF INTEREST}

No potential conflict of interest relevant to this article was reported.

\section{AUTHOR CONTRIBUTIONS}

Conception or design: Y.J.P., S.W.C. Acquisition, analysis, or interpretation of data: S.K., S.K.K., H.S.C., M.J.K. Drafting the work or revising: S.K. Final approval of the manuscript: S.K., Y.J.P., D.J.P., S.W.C.

\section{ORCID}

Sunyoung Kang https://orcid.org/0000-0002-7705-6614

Sun Wook Cho https://orcid.org/0000-0002-7394-3830

\section{REFERENCES}

1. Haugen BR, Alexander EK, Bible KC, Doherty GM, Mandel SJ, Nikiforov YE, et al. 2015 American Thyroid Association management guidelines for adult patients with thyroid nodules and differentiated thyroid cancer: the American Thyroid Association guidelines task force on thyroid nodules and differentiated thyroid cancer. Thyroid 2016;26:1-133.

2. Cibas ES, Ali SZ. The 2017 Bethesda system for reporting thyroid cytopathology. Thyroid 2017;27:1341-6.

3. Gharib H, Papini E, Garber JR, Duick DS, Harrell RM, Hegedus L, et al. American Association of Clinical Endocrinologists, American College of Endocrinology, and Associazione Medici Endocrinologi medical guidelines for clinical practice for the diagnosis and management of thyroid nodules: 2016 update. Endocr Pract 2016;22:622-39.

4. Hong MJ, Na DG, Baek JH, Sung JY, Kim JH. Cytology-ultrasonography risk-stratification scoring system based on fine-needle aspiration cytology and the Korean-Thyroid Imaging Reporting and Data System. Thyroid 2017;27:953-9. 
5. Ianni F, Campanella P, Rota CA, Prete A, Castellino L, Pontecorvi A, et al. A meta-analysis-derived proposal for a clinical, ultrasonographic, and cytological scoring system to evaluate thyroid nodules: the "CUT" score. Endocrine 2016; 52:313-21.

6. Lee SW, Lee HJ, Kim HJ, Lee J, Park JY, Kim SH, et al. Combined categorical reporting systems of US and cytology findings for thyroid nodules: guidance on repeat fine-needle aspiration cytology. Radiology 2013;266:956-63.

7. Tessler FN, Middleton WD, Grant EG, Hoang JK, Berland LL, Teefey SA, et al. ACR Thyroid Imaging, Reporting and Data System (TI-RADS): white paper of the ACR TI-RADS committee. J Am Coll Radiol 2017;14:587-95.

8. Russ G, Bonnema SJ, Erdogan MF, Durante C, Ngu R, Leenhardt L. European Thyroid Association guidelines for ultrasound malignancy risk stratification of thyroid nodules in adults: the EU-TIRADS. Eur Thyroid J 2017;6:225-37.

9. Shin JH, Baek JH, Chung J, Ha EJ, Kim JH, Lee YH, et al. Ultrasonography diagnosis and imaging-based management of thyroid nodules: revised Korean Society of Thyroid Radiology consensus statement and recommendations. Korean J Radiol 2016;17:370-95.

10. Xu T, Wu Y, Wu RX, Zhang YZ, Gu JY, Ye XH, et al. Validation and comparison of three newly-released Thyroid Imaging Reporting and Data Systems for cancer risk determination. Endocrine 2019;64:299-307.

11. Grani G, Lamartina L, Ascoli V, Bosco D, Biffoni M, Giacomelli L, et al. Reducing the number of unnecessary thyroid biopsies while improving diagnostic accuracy: toward the "right" TIRADS. J Clin Endocrinol Metab 2019;104:95102.

12. Nikiforov YE, Seethala RR, Tallini G, Baloch ZW, Basolo F, Thompson LD, et al. Nomenclature revision for encapsulated follicular variant of papillary thyroid carcinoma: a paradigm shift to reduce overtreatment of indolent tumors. JAMA Oncol 2016;2:1023-9.

13. Maletta F, Massa F, Torregrossa L, Duregon E, Casadei GP, Basolo F, et al. Cytological features of "noninvasive follicular thyroid neoplasm with papillary-like nuclear features" and their correlation with tumor histology. Hum Pathol 2016;54:134-42.

14. Layfield LJ, Baloch ZW, Esebua M, Kannuswamy R, Schmidt RL. Impact of the reclassification of the non-invasive follicular variant of papillary carcinoma as benign on the malignancy risk of the Bethesda system for reporting thyroid cytopathology: a meta-analysis study. Acta Cytol
2017;61:187-93.

15. Yoo WS, Ahn HY, Ahn HS, Chung YJ, Kim HS, Cho BY, et al. Malignancy rate of Bethesda category III thyroid nodules according to ultrasound risk stratification system and cytological subtype. Medicine (Baltimore) 2020;99:e18780.

16. Lim JX, Nga ME, Chan DK, Tan WB, Parameswaran R, Ngiam KY. Subclassification of Bethesda atypical and follicular neoplasm categories according to nuclear and architectural atypia improves discrimination of thyroid malignancy risk. Thyroid 2018;28:511-21.

17. Gao LY, Wang Y, Jiang YX, Yang X, Liu RY, Xi XH, et al. Ultrasound is helpful to differentiate Bethesda class III thyroid nodules: a PRISMA-compliant systematic review and meta-analysis. Medicine (Baltimore) 2017;96:e6564.

18. Lee JH, Han K, Kim EK, Moon HJ, Yoon JH, Park VY, et al. Risk stratification of thyroid nodules with atypia of undetermined significance/follicular lesion of undetermined significance (AUS/FLUS) cytology using ultrasonography patterns defined by the 2015 ATA Guidelines. Ann Otol Rhinol Laryngol 2017;126:625-33.

19. Ulisse S, Bosco D, Nardi F, Nesca A, D’Armiento E, Guglielmino $\mathrm{V}$, et al. Thyroid imaging reporting and data system score combined with the new Italian classification for thyroid cytology improves the clinical management of indeterminate nodules. Int J Endocrinol 2017;2017:9692304.

20. Trimboli P, Fulciniti F, Zilioli V, Ceriani L, Giovanella L. Accuracy of international ultrasound risk stratification systems in thyroid lesions cytologically classified as indeterminate. Diagn Cytopathol 2017;45:113-7.

21. Trimboli P, Deandrea M, Mormile A, Ceriani L, Garino F, Limone PP, et al. American Thyroid Association ultrasound system for the initial assessment of thyroid nodules: use in stratifying the risk of malignancy of indeterminate lesions. Head Neck 2018;40:722-7.

22. Maia FF, Matos PS, Pavin EJ, Zantut-Wittmann DE. Thyroid imaging reporting and data system score combined with Bethesda system for malignancy risk stratification in thyroid nodules with indeterminate results on cytology. Clin Endocrinol (Oxf) 2015;82:439-44.

23. Grani G, Lamartina L, Ascoli V, Bosco D, Nardi F, D’Ambrosio F, et al. Ultrasonography scoring systems can rule out malignancy in cytologically indeterminate thyroid nodules. Endocrine 2017;57:256-61.

24. Słowinska-Klencka D, Wysocka-Konieczna K, Klencki M, Popowicz B. Diagnostic value of six Thyroid Imaging Reporting and Data Systems (TIRADS) in cytologically equiv- 
ocal thyroid nodules. J Clin Med 2020;9:2281.

25. Jeh SK, Jung SL, Kim BS, Lee YS. Evaluating the degree of conformity of papillary carcinoma and follicular carcinoma to the reported ultrasonographic findings of malignant thyroid tumor. Korean J Radiol 2007;8:192-7.

26. Kim DS, Kim JH, Na DG, Park SH, Kim E, Chang KH, et al. Sonographic features of follicular variant papillary thyroid carcinomas in comparison with conventional papillary thyroid carcinomas. J Ultrasound Med 2009;28:1685-92.

27. Pusztaszeri M, Auger M. Update on the cytologic features of papillary thyroid carcinoma variants. Diagn Cytopathol 2017;45:714-30.

28. Ooi LY, Nga ME. Atypia of undetermined significance/follicular lesion of undetermined significance: Asian vs. nonAsian practice, and the Singapore experience. Gland Surg 2020;9:1764-87.

29. Saglietti C, Piana S, La Rosa S, Bongiovanni M. Hyalinizing trabecular tumour of the thyroid: fine-needle aspiration cytological diagnosis and correlation with histology. J Clin
Pathol 2017;70:641-7.

30. Evenson A, Mowschenson P, Wang H, Connolly J, Mendrinos S, Parangi S, et al. Hyalinizing trabecular adenoma: an uncommon thyroid tumor frequently misdiagnosed as papillary or medullary thyroid carcinoma. Am J Surg 2007;193: 707-12.

31. Howard BE, Gnagi SH, Ocal IT, Hinni ML. Hyalinizing trabecular tumor masquerading as papillary thyroid carcinoma on fine-needle aspiration. ORL J Otorhinolaryngol Relat Spec 2013;75:309-13.

32. Jang H, Park CK, Son EJ, Kim EK, Kwak JY, Moon HJ, et al. Hyalinizing trabecular tumor of the thyroid: diagnosis of a rare tumor using ultrasonography, cytology, and intraoperative frozen sections. Ultrasonography 2016;35:131-9.

33. Na DG, Kim DS, Kim SJ, Ryoo JW, Jung SL. Thyroid nodules with isolated macrocalcification: malignancy risk and diagnostic efficacy of fine-needle aspiration and core needle biopsy. Ultrasonography 2016;35:212-9. 